\title{
Lessons from the Milky Way: the Kapteyn Universe
}

\author{
P.C. van der Kruit
}

\begin{abstract}
Jacobus Cornelius Kapteyn (1851-1922) presented a model for the distribution of stars in space together with a dynamical interpretation in terms of an equilibrium between the gravitational field of the stars and their random motion and rotation. In the vertical direction Kapteyn's results are substantially correct. Usually the Kapteyn Universe is described as being flawed due to neglect of interstellar absorption. Kapteyn was led to adopt this on the basis of widely accepted evidence by Shapley on an absence of reddening of stars in globular clusters. But another, equally important misconception was Kapteyn's interpretation of the two Star Streams as manifestations of two groups of stars rotating around a center in opposite directions. This was supported by the observation of very different mixes in stellar types in the two streams. Had Kapteyn adopted the absorption as he himself had determined it he would not have been able to arrive at a consistent picture.
\end{abstract}

\section{Introduction}

In 1920 Jacobus C. Kapteyn, together with his student and successor Pieter van Rhijn, published his well-known model for the distribution of stars in space, [7], and a year later presentee a description of its 'mechanics', providing a consistent explanation of how the system could be in equilibrium by a precise balance between the gravitational force of the stars and their random motions and organized rotation, [6]. Ten years later the vertical dynamics was proved substantially correct, but in the plane of the Milky Way the discoveries of differential rotation and interstellar extinction had shown the Kapteyn Universe to be completely wrong. Jan Hendrik Oort, who although completing his thesis after his death always considered himself a student of Kapteyn, played a major role in this with his discovery of Galactic rotation and the introduction of the 'Oort constants' in 1928, [9] and extended Kapteyn's

Kapteyn Astronomical Institute, University of Groningen, P.O. Box 800, 9700AV Groningen, the Netherlandse-mail: vdkruiteastro.rug.nl 
work in the vertical direction with the 'Oort limit' in 1932, [10]. Oort obtained his doctorate under van Rhijn in May 1926 on a study of stars of high velocity and accepted in November of that year a position as 'privaat-docent' in Leiden with a public lecture on Non-light emitting matter in the Stellar System. This was only four years after Kapteyn's demise and preceded his discovery of Galactic rotation by almost two years. In this lecture, of which I provided a translation in Appendix A of a Legacy volume on Kapteyn, [16], he concluded that the 'least contrived' solution to the disparity between Shapley's system of globular clusters and the Kapteyn Universe (Fig. 1) was the assumption of an absorption of light in space. Why had this not been anticipated by Kapteyn? What would have happened had Kapteyn included an absorption correction in his modeling?

\section{The arrangement of stars in space}

Kapteyn had from his earliest research efforts had an interest in the spatial distribution of stars. Distances were of prime importance to this problem. His inaugural lecture on the occasion of his appointment of professor in Groningen in 1878, had the title The parallaxes of the fixed stars. And early on in his career he did some remarkably accurate measurements of trigonometric parallaxes of stars from differential timing measurements of meridian passages, using the Leiden meridian circle. He had not succeeded in securing funds for his own observatory in Groningen in spite of positive support from his university; Leiden and Utrecht opposed the founding of a third, competing observatory in the Netherlands. The stars in Kapteyn's sample were selected as probably nearby on the basis of large proper motion. It is amazing that he was able to measure parallaxes with this method, since for a distance of $10 \mathrm{pc}$ the effect of the annual parallax on the meridian passage is -depending on declination- a few hundredths of a second of time.

Kapteyn devised a clever method to derive absolute declinations, free from systematic errors due to atmospheric refraction and telescope flexure. For this he only measured differences in zenith angles of two stars at that crossed the meridian at about the same time at similar zenith angles on either side of the zenith, azimuths and times of passages through the prime vertical. In 1884, he found David Gill at the Cape Observatory interested in undertaking such a project. Being struck by the possibilities of photographic plates to register star images, Gill decided on the production of the Cape Photographic Durchmusterung and lured Kapteyn in offering his services to measure the plates. To do this Kapteyn invented his 'parallactic' method in which a small telescope at the focal distance from the plate could be used to directly read off celestial coordinates on its axes. This experience and his advisory role in the Carte $\mathrm{du}$ Ciel, caused Kapteyn to undertake extensive photographic work on parallaxes and proper motions, using plates taken for him by Anders Donner of Helsinborg.

He quickly realized that stars were too distant for direct parallax measurements on a grand scale and resolved to use the motion of the Sun with respect to nearby 


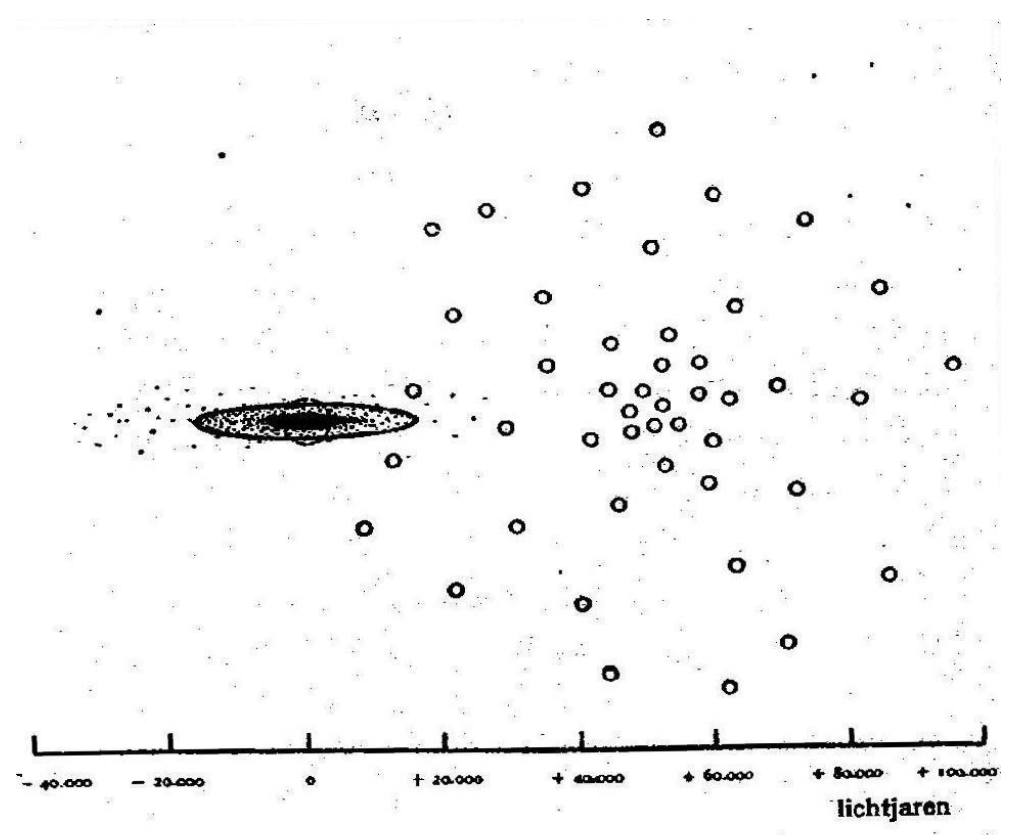

Fig. 1 Oort's illustration of the discrepancy of the Kapteyn Universe and Shapley's system of globular clusters. It appeared in de Sitter;s book 'Kosmos'.

stars (which after all is about four radii of the Earth's orbit per year). To determine the arrangement of stars in space from star counts as a function of apparent magnitude and proper motion, he developed with the help of his mathematics brother Willem the necessary mathematical techniques, making three assumptions. (1) The 'luminosity curve', the distribution of absolute magnitudes, was the same everywhere, (2) there was no preferred direction among the motions of the stars in space, and (3) there was no absorption of starlight.

\section{Star Streams}

However, around 1902 Kapteyn came to the realization that stars did not move completely randomly, but that there were two preferred directions in space, corresponding to positions, 'vertices', about $100^{\circ}$ apart on the sky. When these were corrected for the solar velocity, they were found to be roughly opposite and in the Milky Way (and in agreement with our current understanding, roughly in the direction of the Galactic center and anti-center). To Kapteyn this suggested the existence of two Star Streams. He first announced this in 1904 at a large astronomical congress during the Louisiana Purchase Exhibition in St. Louis, and at the meeting of the British 
Association in Cape Town the next year. It was almost instantly accepted, undoubtedly helped by quick confirmation of the results by other astronomers, notably a young Arthur Stanley Eddington, who had just graduated from Cambridge. The two streams moved at a relative velocity, determined from radial velocities, of order 40 $\mathrm{km} / \mathrm{s}$.

Considering the evidence and data available, this discovery is one of great skill. And observationally it still stands. However, it did not take long before Karl Schwarzschild came up with what we now to be the correct explanation, namely that of an an-isotropic velocity distribution, the directions of the Star Streams corresponding to the long axis of the velocity ellipsoid. Kapteyn was of course aware of this possible explanation, but all his life he believed in his own interpretation of two opposite streams. And he had good reasons for this. Throughout his studies the data available strongly suggested that the composition of the two streams was very different in terms of spectral types of the stars they contained. And Kapteyn was in good company; at the time he made his 'first attempt' to determine the distribution of stars in space, Eddington also favored Kapteyn's interpretation over Schwarzschild's.

\section{Selected Areas}

What was needed was large samples of stars with well-determined magnitudes and proper motions. This could in principle be turned into mean parallaxes as a function of apparent magnitude and proper motions. Together these could then be used to find the apex of the solar motion and the vertices of the streams. Radial velocities were necessary to find the magnitude of the Sun's velocity in space and spectra the types of stars involved for a determination of the mix of ages. Kapteyn interpreted the Main Sequence as an age sequence, blue stars being young. Trigonometric parallaxes were most welcome. Realizing that one needed to go to faint magnitudes, Kapteyn proposed an concerted effort by many observatories by restricting to small areas (size depending on star surface density on the sky), numbering 206 distributed regularly over the sky, for which of as many stars as possible as many parameters as possible would be observed. It is tribute to Kapteyn's diplomatic skills and international standing that he succeeded in bringing this international coordination about.

Edward Pickering at Harvard executed a Durchmusterung of Selected Areas across the whole sky. He agreed to this only after Kapteyn had extended the Plan to include next to the 'Systematic Plan' a 'Special Plan' proposed by Pickering that concentrated on areas in the Milky Way. Plates taken were sent to Groningen to provide positions and magnitudes. This work resulted in the Harvard-Groningen Durchmusterungs that provided positions and magnitudes down to $\mathrm{m}=16$ or so in all Selected Areas. This was published between 1918 and 1924, and was at least partly available for the Kapteyn \& van Rhijn analysis. But that was not deep enough. Crucial to Kapteyn's program was the adoption by George Ellery Hale of the Plan as the prime observational program for his new 60-inch telescope at Mount Wilson. 
From 1908 onwards plates were taken for all northern Selected Areas (numbers 1 through 139), the result of which only appeared in print in 1930. Even preliminary work with these data could only be performed after Kapteyn's death. Most of the work was done by Frederick Seares or under his direction. Walter Adams used the 60 -inch for spectroscopic work, in particular to determine radial velocities.

Hale's adoption of the Plan of Selected Areas for the 60-inch required in his view the personal involvement of Kapteyn and the latter was in 1908, when the telescope became operational, appointed as research associate of the Carnegie Institution. Kapteyn then started to make annual visits to Mount Wilson until World War I prevented him from returning after 1914. He, however, remained research associate until his death.

\section{The Kapteyn Universe}

The analysis in Kapteyn \& van Rhijn (1920), [7], was preliminary. 'Now that, after so many years of preparation, our data seem at last to be sufficient for the purpose, we have been unable to restrain our curiosity and have resolved to carry through completely a small part of the work .... From their data, and average parallaxes of stars as a function of magnitude and proper motion determined by van Rhijn, [17], they constructed a 'luminosity curve', see Fig.2, which was assumed to be the same everywhere in space.

Using mathematical methods partly developed by Karl Schwarzschild, they solved the counts by transforming these into a density of stars as a function of distance. This process was done for four ranges of latitude, $0^{\circ}, 30^{\circ}, 60^{\circ}$ and $90^{\circ}$. The (preliminary) solution only fitted to densities as a function of distance from the Sun, so effectively assumed that the Sun was at or close to the symmetry point of the system. The result found by Kapteyn and van Rhijn is in the top picture of Fig. 4

With this result Kapteyn could do what he long had been preparing for and that was to study the 'mechanics'. He assumed that the system was in equilibrium. In his 1922 paper, [6], he first assumed that the equi-density surfaces were ellipsoids (see Fig. 3. bottom). He did this so that he could calculate relatively straightforwardly the potential assuming that the star distribution and that of the density of gravitating matter was the same. He used a vertical mean velocity of stars of $10 \mathrm{~km} / \mathrm{sec}$ (which corresponds to a velocity dispersion of $12 \mathrm{~km} / \mathrm{sec}$ ). In the vertical direction he then found that the system was in equilibrium if the mass of an average star was between 1.4 and 2.2 solar masses. The average measured mass of a binary was 1.6 in these units, so that if all stars were binaries there would be no need to invoke 'dark matter in the Universe'. At least, 'this mass cannot be excessive'. This is a first application of what we now call stellar or galactic dynamics. In modern terms, using the isothermal sheet description of van der Kruit \& Searle, [15], Kapteyn's density distribution is fitted pretty well with $z_{\circ}=650 \mathrm{pc}$, rather close to the $700 \mathrm{pc}$ or so we currently use. His velocity dispersion is a bit low compared to the modern $17 \mathrm{~km} / \mathrm{sec}$ or so.

For the horizontal direction, equilibrium could only be achieved if there were some rotation. To Kapteyn it seemed natural that this was the phenomenon of his 


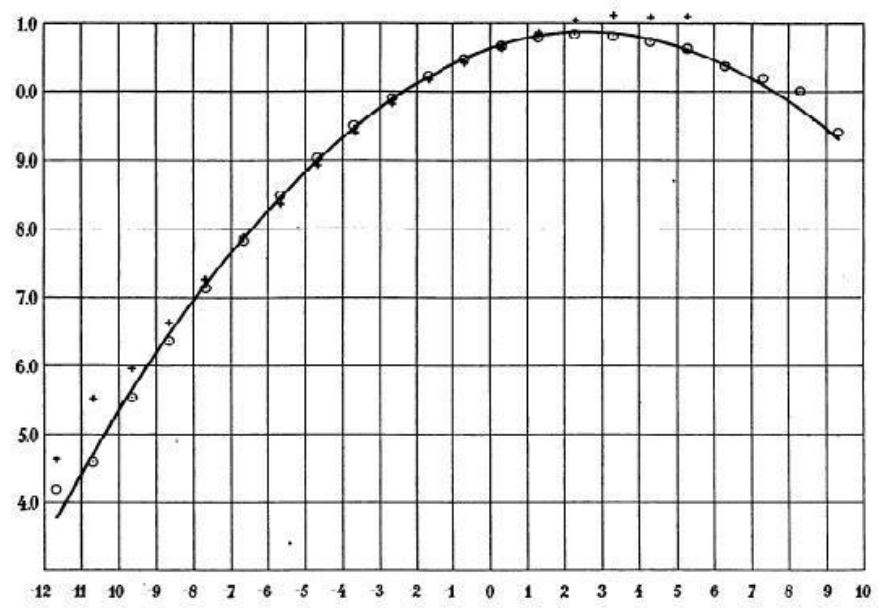

Fig. 2 The 'luminosity curve' derived bi Kapteyn and van Rhijn in [7]. Because Kapteyn had grudgingly accepted the term parsec, he felt obliged to redefine absolute magnitudes to a distance of $1 \mathrm{pc}$.

Star Streams; about half the stars rotating in one direction and the other in the opposite one. The direction towards the (rotation) center would be perpendicular to the streams and could then be in only two directions, one of which was Carina and indeed there the Milky Way is very bright. The relative velocity was $40 \mathrm{~km} / \mathrm{sec}$, so it seemed natural to assume that the rotation velocity was $20 \mathrm{~km} / \mathrm{sec}$. Indeed, when he estimated what it had to be with the mass densities that he derived from the vertical equilibrium he found $18-20 \mathrm{~km} / \mathrm{sec}$ ! But for this to work, the Sun had to be away from the center. Kapteyn argued that this implied a distance up to $1.5 \mathrm{kpc}$, but for reasons having to do with details of the solutions in the density distributions he settled for $650 \mathrm{pc}$. For the vertical displacement he assumed $38 \mathrm{pc}$, based upon a study of the distribution of Cepheids by his son-in-law Ejnar Hertzsprung. The resulting position of the Sun has been indicated with the ' $S$ ' near the small circle in Fig. 3 . bottom.

So we see, that the adoption of this model by Kapteyn had also a dynamical basis, partly correct (in the vertical direction), partly incorrect (due to his interpretation of the Star Streams). But it was ingenious and his introduction of a dynamical framework farsighted.

\section{Kapteyn's studies of absorption}

Kapteyn had always worried about absorption. Over his career he wrote four papers on this subject, one in the Astronomical Journal, three in the Astrophysical Journal. In 1903, Pickering, [11], had found that the star ratio (the ratio of numbers 


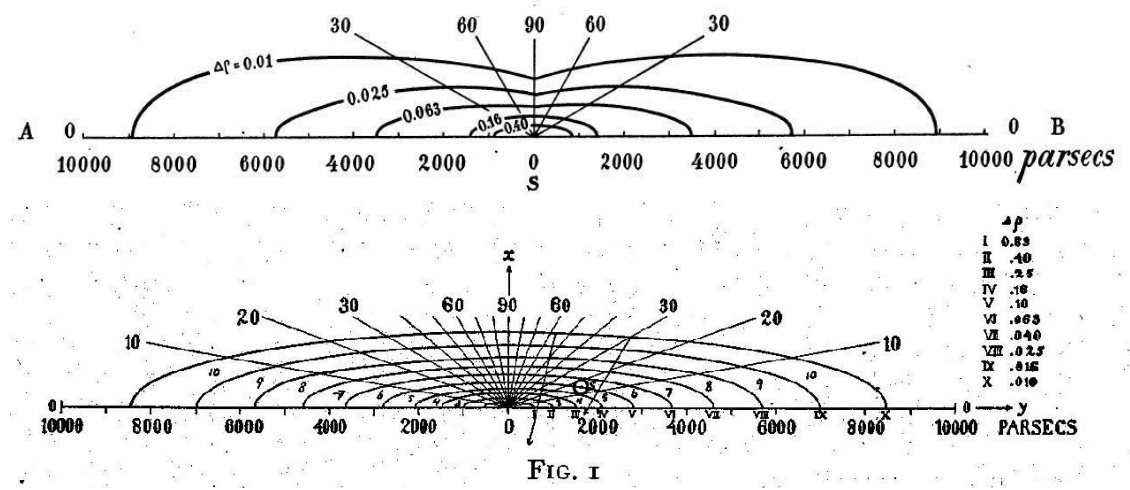

Fig. 3 On top the distributions of stars in space as determined by Kapteyn and van Rhijn (1920). The solution involves a dependence only with distance from the Sun at latitudes $0^{\circ}, 30^{\circ}, 60^{\circ}$ and $90^{\circ}$. The bottom illustration is from Kapteyn (1922), where he fitted ellipsoids to the densities to facilitate computation of the gravitational potential. His interpretation of the Star Streams as two opposite rotations led him to adopt a position of the Sun 650 pa away from the center and on the basis of Hertzsprung study of the distribution of Cepheids on the sky at $38 \mathrm{pc}$ from the plane. The Sun is at the circle designated ' $\mathrm{S}$ '.

of stars between two consecutive magnitudes) deviated from the 'theoretical' value for a uniform distribution of stars in space. He suggested that there was significant absorption, amounting in Kapteyn's notation to 0.18 magnitudes per unit distance. Since this unit is the distance corresponding to a parallax of 0 "! 1 , this is an extremely large amount. Moreover, in 1904 George Comstock found that the mean proper motion of stars at a fixed magnitude depended on Galactic latitude (the smallest in the Milky Way), which also would indicate interstellar absorption, [1]. In 1904, Kapteyn, [2], investigated these claims, showing how sensitively a small amount of extinction would change the inferred stellar distributions. He used the slope of the luminosity curve as a fiducial point to link to observed counts and concluded that some absorption indeed was likely. But he objected against the (extremely large) values of Comstock, since these would put the Sun in a very special place, namely near an extreme minimum in the star density.

In 1909 Kapteyn wrote two papers in the Astrophysical Journal on absorption, [3], [4]. There was not much convincing evidence for absorption by gas (in stellar spectra), but he speculated that absorption by dust would result in a reddening of the starlight. He found values corresponding to 0.001 magnitude per parsec in the photographic band (and 0.0005 in the visual), which at present is a bit small but not far off the mark. In a paper in 1914, in which he returned to the issue by reviewing its status at the time, he reaffirmed this but concluded that from observational data it could not be proven that this was not be the result of a correlation of color with absolute magnitude, [5].

The deciding piece of evidence, however, was Harlow Shapley's result in 1916, [12], that the colors of the stars in the globular cluster M13 (at $10 \mathrm{kpc}$ ) should be 2.5 magnitudes redder than they were observed to be if Kapteyn's value for the absorp- 
tion were adopted. Space was, according to Shapley, transparent. This must have convinced Kapteyn (and with him others) that absorption was only of secondary importance.

Shapley went on to map the distribution of globular clusters and found them to be scattered over a somewhat ellipsoidal volume with the center in the direction of Sagittarius, roughly perpendicular to Kapteyn's center, and at a distance of $20 \mathrm{kpc}$, see [13] and Fig. 1 therein. Shapley's distance to the center was too large, as we now know, to some extent also as a result of absorption since many clusters are at a somewhat low latitude.

What did Kapteyn himself think of Shapley's result? In one of his last papers, written with van Rhijn in 1922, [8], it was argued that Shapley's distances were too large because the local calibrating Cepheids of short periods had high proper motions and therefore had to be near and thus fainter than Shapley's variables in the globular clusters. The longer period variables were giving a different result and Kapteyn and van Rhijn argued that this result was inferior. It was supported by work of Kapteyn's student Schouten, who derived distances to globular clusters by adopting the same luminosity curve as locally. This of course ignored the fact that locally many stars are dwarfs, while all stars used in the clusters were giants.

\section{What if Kapteyn had not neglected absorption?}

I have been asked this question regularly. Obviously we do not know for sure. Kapteyn presented his model for the Sidereal System less than a year before his death. It was in accord with current knowledge and provided a consistent description. As a conclusion of this contribution I quote from the draft of my soon (later this year) to appear biography Jacobus Cornelius Kapteyn: Born investigator of the Heavens, [14].

Finally, one might wonder what would have happened had Kapteyn adopted interstellar absorption. That would have been much in violation of Shapley's result and Kapteyn would for some reason or the other have had to assume it depended on latitude. He did find values for extinction in his earlier work that in hindsight are not unreasonable, but he derived that from relatively bright stars all over the sky. No latitude dependence was evident, and it seems unlikely that he would have detected any had he looked for it.

Had Kapteyn applied an absorption correction he would have found a significantly flatter vertical profile of star densities, which would have lowered the deduced average mass of a star, destroying the dynamical consistency that he found. Similarly, a much flatter radial density profile would have made the picture of the rotation as revealed by the star streams more problematic. If it would have had a central point of maximum density it would be further away than in the Kapteyn a universe and a rotation speed of $20 \mathrm{~km} / \mathrm{sec}$ as revealed by the Star Streams would have to be linked to a rotation around a more distant center. As it was, Kapteyn's solution of such a rotation around a center a half to one kiloparsec away dynamically 

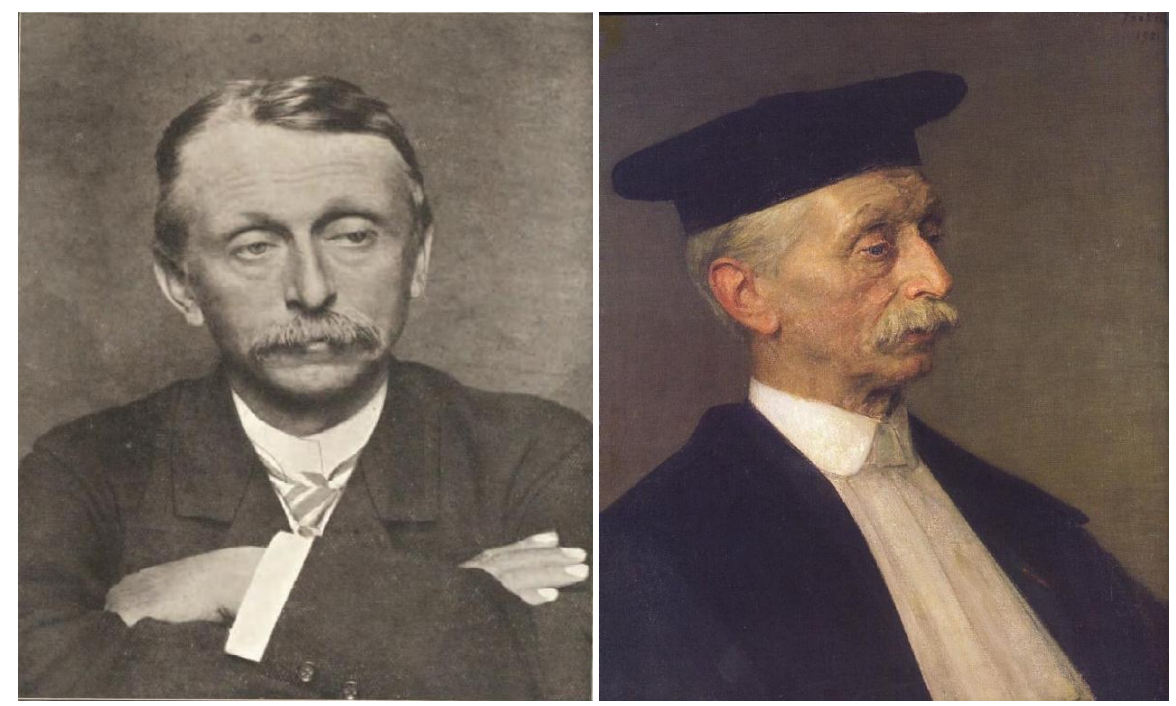

Fig. 4 Jacobus Cornelius Kapteyn (1851-1922) at age 40 and 70.

made sense, and it is not obvious that such a picture could be constructed with so small a rotation in an extended stellar system. [...]

So there were at least two fundamental issues involved. Kapteyn's Universe was not only false because his assumption of transparent space turned out incorrect, but also his conviction that the star streams did not result from an anisotropy in the random stellar motions was essential for his arriving at a consistent picture. The Kapteyn Universe was built on both pillars and both eventually had to be replaced.

Kapteyn's picture of the Sidereal System was difficult to avoid. Lodewijk Woltjer in [16] referred to it as 'Kapteyn's unfortunate Universe', on account of 'the unlucky moment at which Kapteyn presented his Universe and to the perhaps somewhat unphysical interpretation he gave to the two 'Star Streams', which had contributed so much to his fame'. It is more uncertain to speculate what would have happened had Kapteyn lived a decade or so longer and would have been around when Galactic differential rotation and interstellar absorption were established.

\section{Lessons?}

My chapter in [16] started as follows: 'Around 1990 the Kapteyn Astronomical Institute decided to start a preprint series and we chose to display on the covers a picture of Kapteyn. Some of us were told privately and discretely by a few of our colleagues abroad (mostly in the U.S.) that is was inappropriate to use this picture. After all, Kapteyn had been proven wrong in almost all respects; his work on interstellar absorption was shown to seriously underestimate the effect, while his model of the distribution of stars in space 
and his description of the kinematics and dynamics in terms of circular motions in two star streams was also incorrect. A consensus among our staff eventually led to the replacement of that picture by a 'more current' one; I regret having not more strongly opposed that then.' It was the picture at the right in Fig. 4, and I still do regret this.

Owen Gingerich, in the same volume, wrote: 'For American college students studying introductory astronomy, the chances are about even that they will encounter the name of Kapteyn. The distinguished astronomer of Groningen is mentioned in seven of ten recent textbooks that I examined, always associated with the Kapteyn Universe, and invariably as a foil for Shapley's larger and more modern conception of the Milky Way Galaxy. While the presentation in American textbooks places Kapteyn and Shapley as intellectual rivals and their systems as totally antithetical, the historical reality is much different.'

So, two lesson are: 'Don't take historical introductions in elementary textbooks too seriously. And be warned that your colleagues may very well have done that anyway'.

Acknowledgement. I am very grateful to Ken Freeman for presenting the review when, due to family affairs, I had to cancel my participation to the conference at the last moment.

\section{References}

1. Comstock, G.C. (1904), A.J. 24, 43.

2. Kapteyn, J.C. (1904), A.J. 24, 115.

3. Kapteyn, J.C. (1909a), Ap.J. 29, 46.

4. Kapteyn, J.C. (1909b), Ap.J. 30, 284.

5. Kapteyn, J.C. (1914), Ap.J. 40, 187.

6. Kapteyn, J.C. (1922), Ap.J. 55, 302.

7. Kapteyn, J.C. \& van Rhijn, P.J. (1920), Ap.J. 52, 23.

8. Kapteyn, J.C. \& van Rhijn, P.J. (1922), B.A.N. 1, 37.

9. Oort, J.H. (1928), B.A.N. 4, 269.

10. Oort, J.H. (1932), B.A.N. 6, 249.

11. Pickering, E.C. (1903), ANn. HAR. 48, 149.

12. Shapley, H. (1916), Proc. Nat Acad. Sci. 2, 12.

13. Shapley, H. (1919), Ap.J. 49, 311.

14. van der Kruit, P.C (2014), Jacobus Cornelius Kapteyn: Born investigator of the Heavens, Springer, in preparation.

15. van der Kruit, P.C. \& Searle, L. (1981), A.\&A. 95, 105.

16. van der Kruit, P.C. \& van Berkel, K. (2000), The Legacy of J. C. Kapteyn. Studies on Kapteyn and the development of modern astronomy, Springer, ISBN 0-7923-6393-0.

17. van Rhijn, P.J. (1920), Publ. Astron. Lab. Groningen, 30. 